\title{
Economic Burden of Treatment-Resistant Depression in Privately Insured U.S. Patients with Physical Conditions
}

\author{
Maryia Zhdanava, MA; Harsh Kuvadia, MA; Kruti Joshi, MPH; Ella Daly, MD; Dominic Pilon, MA; \\ Carmine Rossi, PhD; Laura Morrison, MSc; Patrick Lefebvre, MA; and Craig Nelson, MD
}

\begin{abstract}
BACKGROUND: Little is known about the economic burden of treatmentresistant depression (TRD) in patients with physical conditions.

OBJECTIVE: To assess health care resource utilization (HRU) and costs, work loss days, and related costs in patients with TRD and physical conditions versus patients with the same conditions and non-TRD major depressive disorder (MDD) or without MDD.
\end{abstract}

METHODS: Adults aged $<65$ years with MDD treated with antidepressants were identified in the OptumHealth Care Solutions database (July 2009March 2017). Patients who received a diagnosis of MDD and initiated a third antidepressant regimen (index date) after 2 regimens of adequate dose and duration were defined as having TRD. Patients with non-TRD MDD and without MDD were assigned a random index date. Patients with $<6$ months of continuous health plan eligibility pre- or post-index; a diagnosis of psychosis, schizophrenia, bipolar disorder/mania, dementia, and developmental disorders; and/or no baseline physical conditions (cardiovascular, metabolic, and respiratory disease or cancer) were excluded. Patients with TRD were matched 1:1 to each of the non-TRD MDD and non-MDD cohorts based on propensity scores. Per patient per year $\mathrm{HRU}$, costs, and work loss outcomes were compared up to 24 months postindex date using negative binominal and ordinary least square regressions. RESULTS: A total of 2,317 patients with TRD (mean age, 47.6 years; $63.1 \%$, female; mean follow-up, 19.7 months) had $\geq 1$ co-occurring key physical condition (cardiovascular, 52.5\%; metabolic, $48.2 \%$; respiratory, $16.4 \%$; and cancer, $9.5 \%$ ). Relative to non-TRD MDD and non-MDD cohorts, respectively, patients with TRD had $46 \%$ and $235 \%$ more inpatient admissions, $28 \%$ and $128 \%$ more emergency department visits, and $53 \%$ and $155 \%$ more outpatient visits (all $P<0.05$ ). Health care costs were $\$ 22,541$ in the TRD cohort, $\$ 17,450$ in the non-TRD MDD cohort, and $\$ 10,047$ in the non-MDD cohort, yielding cost differences of $\$ 5,091$ (vs. non-TRD MDD) and $\$ 12,494$ (vs. non-MDD; all $P<0.01$ ). In patients with work loss data available ( $n=278 /$ cohort), those with TRD had 2.0 and 2.9 times more work loss as well as $\$ 8,676$ and $\$ 10,323$ higher work loss costs relative to those with non-TRD MDD and without MDD, respectively (all $P<0.001$ ).

CONCLUSIONS: In patients with physical conditions, those with TRD had higher HRU and health care costs, work loss days, and associated costs compared with non-TRD MDD and non-MDD cohorts.

J Manag Care Spec Pharm. 2020;26(8):996-1007

Copyright $@ 2020$, Academy of Managed Care Pharmacy. All rights reserved.

\section{What is already known about this subject}

Approximately a third of patients with major depressive disorder (MDD), who receive pharmacologic treatment, have treatmentresistant depression (TRD). These patients incur significantly higher health care costs than those with treatment-responsive MDD (i.e., non-TRD MDD) and without MDD.

Patients with MDD and, in particular those with TRD, have higher rates of physical conditions than individuals without MDD

Despite the documented interplay between depression and physical health, limited data are available on the health care resource utilization (HRU) and health care costs of patients with TRD and co-occurring physical conditions.

\section{What this study adds}

Patients with TRD and concurrent physical conditions incurred higher all-cause HRU and higher all-cause health care costs compared with patients with the same physical conditions and non-TRD MDD or without MDD.

Medical costs related to physical conditions were similar in patients with MDD regardless of TRD but were significantly higher in patients with TRD versus those without MDD. Thus, MDD may complicate the clinical management of physical conditions or have a negative impact on their course.

The work loss-related costs of patients with TRD were more than 2-fold higher than those of patients with non-TRD MDD and 3-fold higher than those of patients without MDD.

$\mathrm{M}$ ajor depressive disorder (MDD) is a common and incapacitating psychiatric condition with a 12-month prevalence of $7.2 \%$ in the United States in 2018. About $50 \%$ of individuals with MDD are reported to receive pharmacotherapy for their condition. ${ }^{2}$ Among them, approximately one third are estimated to have treatment-resistant depression (TRD), ${ }^{3}$ which is most commonly defined as an absence of response to $\geq 2$ different antidepressant regimens of adequate dose and duration. ${ }^{4}$

The incremental economic burden of patients with TRD relative to patients with treatment-responsive MDD (i.e., nonTRD MDD) or individuals without MDD is substantial across U.S. payers, including commercial health plans, ${ }^{5}$ Medicare, ${ }^{6}$ Medicaid, ${ }^{7,8}$ and Veterans Health Administration. ${ }^{3}$ Specifically, 
commercially insured patients with TRD were reported to have $\$ 6,709$ higher annual costs compared with patients with non-TRD MDD and \$9,917 higher annual costs compared with individuals without MDD. ${ }^{5}$ On a national scale, patients with TRD are estimated to incur a direct health care costs burden of approximately $\$ 30$ billion annually, representing more than $50 \%$ of the overall cost burden of pharmacologically treated MDD. ${ }^{3}$ Furthermore, patients with TRD were shown to exhibit greater work loss-related costs than matched controls with non-TRD MDD and without MDD. ${ }^{5,9}$

Physical conditions often accompany mental illness. At least half of patients with MDD are affected by 1 or more co-occurring physical conditions. ${ }^{10,11}$ Patients with MDD, and in particular those with TRD, have higher rates of comorbidities than individuals without MDD. 5,6,8,12 Moreover, TRD appears to be associated with higher rates of cardiovascular disease, ${ }^{7,13}$ metabolic disorders, ${ }^{12,14-16}$ and respiratory disease ${ }^{13}$ relative to non-TRD MDD. Notably, the severity of depression has been shown to be associated with the presence of comorbidities, ${ }^{17}$ and previous studies found that co-occurring physical conditions are associated with a reduced likelihood of response to antidepressants. ${ }^{10,11}$

Given the interactions between depression and physical health, patients with TRD and co-occurring physical conditions may incur substantially increased health care utilization and higher health care costs. The present study sought to assess health care resource utilization (HRU) and costs, work loss days, and related costs among privately insured U.S. patients with TRD and key physical conditions compared with patients with the same physical conditions who either had non-TRD MDD or did not have MDD.

\section{Methods}

\section{Data Source}

The Optum Health Care Solutions commercial insurance claims database (July 1, 2009-March 31, 2017) was used. This database contains information on 19.1 million privately insured individuals nationwide, including claimant demographics and insurance eligibility; medical and prescription drug claims; and short- and long-term disability claims, salary, and employment status for a subset of patients. This database only contains de-identified data that fully comply with the patient confidentiality requirements of the Health Insurance Portability and Accountability Act. Per Title 45 of Code of Federal Regulations, Part 46.101(b)(4), ${ }^{18}$ the analysis of our study is exempt from institutional review for the following reasons: (a) it is a retrospective analysis of existing data (hence no patient intervention or interaction), and (b) no patientidentifiable information is included in the claims dataset.

\section{Study Design}

A retrospective longitudinal matched cohort study design was used. Patients (between ages 18 and 64 years) with MDD and TRD and key physical conditions including cardiovascular,, 13 metabolic, ${ }^{12,14-16}$ and respiratory diseases ${ }^{13}$ or cancer $^{13}$ (the TRD cohort) were matched and compared with 2 cohorts of patients with the same physical conditions who (a) had non-TRD MDD (the non-TRD MDD cohort) and (b) did not have MDD (the non-MDD cohort). The study period spanned from July 1 , 2009, to March 31, 2017.

For patients in the TRD cohort, the index date was defined as the initiation of a third antidepressant treatment course after absence of response to 2 courses of adequate dose and duration within the same MDD episode. Adequate dose was defined as a starting dose recommended by the American Psychiatric Association treatment guidelines. ${ }^{19}$ Adequate duration was defined as at least 6 weeks of continuous therapy with no gaps longer than 14 days. For patients in the non-TRD MDD cohort, the index date was imputed after the first antidepressant so that the distribution of time elapsed from the first antidepressant to the imputed index date would mimic that observed in the TRD cohort. For patients in the non-MDD cohort, the index date was imputed after the start of continuous insurance eligibility so that the distribution of time elapsed from the start of insurance eligibility to the imputed index date would mimic that observed in the TRD cohort.

Baseline characteristics (including key physical conditions) were evaluated in the 6-month period before the index date, which was defined as the baseline period. Study outcomes were evaluated during the follow-up period. The follow-up period spanned from the index date to the earliest among the following events: (a) the end of continuous eligibility or data availability (March 31, 2017), (b) 2 years post-index date, or (c) among the non-MDD cohort, a diagnosis of MDD or an antidepressant claim.

\section{Definition of Study Cohorts}

Patients included in the TRD and non-TRD MDD cohorts met the following criteria: (a) $\geq 1$ diagnosis of MDD (International Classification of Diseases, Ninth Revision, Clinical Modification [ICD-9-CM]: 296.2x, 296.3x; International Classification of Diseases, Tenth Revision, Clinical Modification [ICD-10-CM]: F32.x [excluding F32.8], F33.x [excluding F33.8]) during the study period; (b) $\geq 1$ antidepressant claim of adequate dose and duration after January 1, 2010; (c) no claims for an antidepressant in the 6 months before the first antidepressant claim; and (d) $\geq 1$ diagnosis for depression (ICD-9-CM: 296.2x, 296.3x, 300.4x, 311.x, 309.0x, or 309.1x; ICD-10-CM: F32.x, F33.x, F34.1, or F43.21) during the period from 6 months before the first antidepressant claim up to 2 years after the index date. 
Those patients included in the TRD cohort were additionally required to start a third antidepressant treatment course after an absence of response to 2 antidepressant courses (including augmentation therapy with anticonvulsant, antipsychotic, lithium, psychostimulant, and thyroid hormone medications) of adequate dose and duration within 2 years of the first antidepressant claim. An absence of response was defined as a switch of an antidepressant ( $<180$ days after the end of the previous treatment), an addition of an antidepressant, or an initiation of an augmentation therapy. ${ }^{5}$ The criteria of $<180$ days between treatment courses and no more than 2 years between the first antidepressant claim and the third antidepressant treatment course helped to ensure that the onset of TRD was identified within the same MDD episode. ${ }^{14}$

Patients included in the non-MDD cohort had neither claims for an antidepressant nor diagnoses for MDD before the index date or within $\geq 6$ months afterward.

Finally, patients in all 3 cohorts met the following criteria: (a) no diagnosis for specific psychiatric conditions (i.e., psychosis, schizophrenia, bipolar disorder/manic depression, dementia, mental retardation, other specific delays in development, and pervasive developmental disorders) during the study period; (b) $\geq 6$ months of continuous insurance eligibility before and after the index date; (c) $\geq 1$ claim for a key physical health condition (i.e., cardiovascular, metabolic, and respiratory disease or cancer) during the baseline period; and (d) aged 18-65 years at the index date.

\section{Outcome Measures}

HRU and health care costs were reported by category, including inpatient, emergency department (ED), outpatient, and other services. Health care cost categories also included medical costs (i.e., sum of inpatient, ED, outpatient, and other costs); pharmacy costs; and total health care costs (sum of medical and pharmacy costs).

Work loss and related costs were evaluated for employees with available work loss information and included paid disability and medical-related absenteeism. Paid disability days and associated costs were obtained from the data directly. Days of medical-related absenteeism were imputed as the length of stay of inpatient admissions, 1 day for each ED visit, or a half a day for each outpatient and other visit, plus days when the patient was eligible for disability, but not yet receiving benefits. Costs of medical-related absenteeism were obtained based on the number of days of medical-related absenteeism and wage data. All outcomes were reported per patient per year; in patients with less than a year of follow-up, outcomes were extrapolated for the full year based on the available data. Costs were expressed in 2017 U.S. dollars and measured from a payer's perspective. Physical condition-related outcomes were defined based on claims with a diagnosis for any of the key physical conditions (i.e., cardiovascular, metabolic, and respiratory diseases or cancer). Additionally, behavioral health-related outcomes were defined based on claims with an ICD-9-CM diagnosis between 290 and 319 (inclusive) or an ICD-10-CM diagnosis between F01 and F99 (inclusive).

\section{Statistical Analysis}

Patients with TRD were matched 1:1 to patients with non-TRD MDD and without MDD based on (a) the availability of work loss data (exact match) and (b) age, sex, year of the index date, geographical region, type of health care plan, and presence of each physical condition (propensity score match). The balance of baseline characteristics between cohorts after matching was assessed with standardized differences $(<10 \%$ indicated balance). HRU and work loss outcomes during the followup period were compared between matched cohorts using unadjusted negative binomial regressions that accounted for the correlation between matched pairs. Results were reported as incidence rate ratios (IRRs) with 95\% confidence intervals (CIs) and $P$ values. Cost outcomes were reported as mean cost differences between matched cohorts, which were estimated using unadjusted ordinary least squares regressions; to account for a nonnormal distribution of cost data, 95\% CIs and $P$ values were obtained from nonparametric bootstrap procedures with 500 replications.

\section{Results}

Of the 7,135 patients with TRD who met study selection criteria, 2,317 (32.5\%) had $\geq 1$ key co-occurring physical condition. Among 45,690 patients with non-TRD MDD who met study inclusion criteria, 13,003 (28.5\%) had $\geq 1$ key co-occurring physical condition. A total of 2,317 patients from the TRD cohort were matched 1:1 to patients with non-TRD MDD and without MDD.

\section{Baseline Characteristics}

After matching, cohorts were well balanced in terms of demographic characteristics and key physical conditions (Table 1). In the 3 cohorts, mean age was 47.3-47.7 years; 62.4\%-64.8\% were female. Mean Quan-Charlson Comorbidity Index was 0.9 in the TRD cohort and 0.8 in the non-TRD MDD and non-MDD cohorts. Between $51.1 \%$ and $52.5 \%$ of patients in each cohort had cardiovascular disease, $47.7 \%$ and $48.2 \%$ had a metabolic disease, $16.0 \%$ and $16.4 \%$ had a respiratory disease, and $9.0 \%$ and $9.9 \%$ had cancer. The annualized mean (median) number of days with outpatient visits related to a key physical condition during the baseline period was 5.5 (4.0) in the TRD cohort, 5.4 (4.0) in the non-TRD MDD cohort, and 4.3 (2.0) in the non-MDD cohort.

Given that cohorts were not matched on the presence of behavioral health conditions, anxiety and substance use disorder (SUD) were more common in the TRD cohort (anxiety, 36.9\%; SUD, 11.4\%) than the non-TRD MDD (anxiety, 23.6\%; 


\section{TABLE 1 Baseline Characteristics in Matched Cohorts ${ }^{a}$}

\begin{tabular}{|c|c|c|c|c|c|}
\hline & $\begin{array}{l}\text { TRD Cohort } \\
\mathrm{n}=2,317\end{array}$ & $\begin{array}{l}\text { Non-TRD MDD Cohort } \\
\qquad \mathbf{n}=2,317\end{array}$ & Std Diff, \% & $\begin{array}{l}\text { Non-MDD Cohort } \\
\quad \mathbf{n}=2,317\end{array}$ & Std Diff, \% \\
\hline Age, years, mean \pm SD (median) & $47.6 \pm 12.0(50)$ & $47.3 \pm 12.3(50)$ & 2.1 & $47.7 \pm 12.0(50)$ & 1.4 \\
\hline Female, n (\%) & $1,463(63.1)$ & $1,501 \quad(64.8)$ & 3.4 & $1,445 \quad(62.4)$ & 1.6 \\
\hline \multicolumn{6}{|l|}{ Year of index date, $n(\%)$} \\
\hline 2010 & $302(13.0)$ & $308(13.3)$ & 0.8 & $334(14.4)$ & 4.0 \\
\hline 2011 & $532(23.0)$ & $565(24.4)$ & 3.4 & $543(23.4)$ & 1.1 \\
\hline 2012 & $423(18.3)$ & $447(19.3)$ & 2.7 & $414 \quad(17.9)$ & 1.0 \\
\hline 2013 & $344(14.8)$ & $318(13.7)$ & 3.2 & $302(13.0)$ & 5.2 \\
\hline 2014 & $295(12.7)$ & $287(12.4)$ & 1.0 & $306(13.2)$ & 1.4 \\
\hline 2015 & $238(10.3)$ & $224 \quad(9.7)$ & 2.0 & $232(10.0)$ & 0.9 \\
\hline 2016 & $183 \quad(7.9)$ & $168 \quad(7.3)$ & 2.4 & $186 \quad(8.0)$ & 0.5 \\
\hline \multicolumn{6}{|l|}{ Geographic region, $\mathbf{n}(\%)$} \\
\hline South & $812(35.0)$ & $824(35.6)$ & 1.1 & $837(36.1)$ & 2.3 \\
\hline Midwest & $578(24.9)$ & $552(23.8)$ & 2.6 & $534(23.0)$ & 4.4 \\
\hline Northeast & $495(21.4)$ & $505 \quad(21.8)$ & 1.0 & $512(22.1)$ & 1.8 \\
\hline West & $303(13.1)$ & $303(13.1)$ & 0 & $305(13.2)$ & 0.3 \\
\hline Unknown & $129 \quad(5.6)$ & $133 \quad(5.7)$ & 0.7 & $129 \quad(5.6)$ & 0 \\
\hline \multicolumn{6}{|l|}{ Type of health care plan, n (\%) } \\
\hline Preferred provider organization & $1,710(73.8)$ & $1,703(73.5)$ & 0.7 & $1,722 \quad(74.3)$ & 1.2 \\
\hline Point of service plan & $320(13.8)$ & $335(14.5)$ & 1.9 & $320(13.8)$ & 0 \\
\hline Indemnity plan (i.e., fee for service) & $236(10.2)$ & $234(10.1)$ & 0.3 & $224 \quad(9.7)$ & 1.7 \\
\hline Other health care plan & $51 \quad(2.2)$ & $45 \quad(1.9)$ & 1.8 & $51 \quad(2.2)$ & 0 \\
\hline \multicolumn{6}{|l|}{ Relationship to health care plan holder, n (\%) } \\
\hline Employee & $1,324(57.1)$ & $1,375(59.3)$ & 4.5 & $1,406(60.7)$ & 7.2 \\
\hline Available work loss data & $278(12.0)$ & $278(12.0)$ & 0 & $278(12.0)$ & 0 \\
\hline Spouse & $805(34.7)$ & $745(32.2)$ & 5.5 & $764(33.0)$ & 3.7 \\
\hline Child & $186 \quad(8.0)$ & $194 \quad(8.4)$ & 1.3 & $144 \quad(6.2)$ & 7.1 \\
\hline Other/unknown & $2 \quad(0.1)$ & $3 \quad(0.1)$ & 1.3 & $3 \quad(0.1)$ & 1.3 \\
\hline Quan-CCI, 21 mean \pm SD (median) & $0.9 \pm 1.3(1)$ & $0.8 \pm 1.3(0)$ & 4.7 & $0.8 \pm 1.2(0)$ & 10.0 \\
\hline \multicolumn{6}{|l|}{ Number of unique agents, mean \pm SD (median) } \\
\hline $\mathrm{AD}$ agents & $1.8 \pm 0.8(2)$ & $1.1 \pm 0.6(1)$ & 104.1 & - & - \\
\hline Psychiatric agents ${ }^{\mathrm{b}}$ & $3.2 \pm 1.5$ (3) & $1.7 \pm 1.1 \quad(1)$ & 116.3 & $0.1 \pm 0.3(0)$ & 289.7 \\
\hline Key physical conditions, n (\%) & $2,317(100.0)$ & $2,317(100.0)$ & 0 & $2,317(100.0)$ & 0 \\
\hline Cardiovascular disease & $1,216(52.5)$ & $1,183 \quad(51.1)$ & 2.9 & $1,206(52.1)$ & 0.9 \\
\hline Metabolic diseases & $1,116(48.2)$ & $1,114(48.1)$ & 0.2 & $1,106 \quad(47.7)$ & 0.9 \\
\hline Respiratory diseases & $381(16.4)$ & $371(16.0)$ & 1.2 & $381(16.4)$ & 0 \\
\hline Cancer & $221 \quad(9.5)$ & $208 \quad(9.0)$ & 1.9 & $229 \quad(9.9)$ & 1.2 \\
\hline Key behavioral health conditions, n (\%) & $995(42.9)$ & $630 \quad(27.2)$ & 33.5 & $(3.5)$ & 105.9 \\
\hline Anxiety & $854(36.9)$ & $546(23.6)$ & 29.3 & (1.9) & 98.8 \\
\hline Substance use disorder & $263(11.4)$ & $124 \quad(5.4)$ & 21.8 & $37 \quad(1.6)$ & 40.4 \\
\hline $\begin{array}{l}\text { Total health care costs, } 2017 \text { USD, } \\
\text { per patient per year, mean } \pm \text { SD (median) }\end{array}$ & $30,039 \pm 70,215(11,271)$ & $20,247 \pm 58,084 \quad(6,021)$ & 15.2 & $12,622 \pm 42,994 \quad(2,827)$ & 29.9 \\
\hline Medical costs & $25,262 \pm 67,882 \quad(6,750)$ & $17,152 \pm 56,192 \quad(3,518)$ & 13.0 & $10,738 \pm 42,063 \quad(1,552)$ & 25.7 \\
\hline Pharmacy costs & $4,777 \pm 12,529 \quad(2,155)$ & $3,094 \pm 9,887 \quad(956)$ & 14.9 & $1,884 \pm 6,514$ & 29.0 \\
\hline $\begin{array}{l}\begin{array}{l}\text { Follow-up time, months, mean } \pm S D \\
\text { (median) }\end{array} \\
\end{array}$ & $19.7 \pm 6.1$ & $20.5 \pm 5.6$ & 13.8 & $19.8 \pm 5.9$ & 2.5 \\
\hline \multicolumn{6}{|c|}{$\begin{array}{l}\text { aPatients were matched on exact matching factors (i.e., availability of work loss data) and the propensity score (the probability of being in the TRD cohort vs. the non-TRD } \\
\text { MDD or non-MDD cohort), modeled using logistic regression adjusted for categoric age, sex, year of the index date, each key physical health condition, geographic region, } \\
\text { and type of health care plan. } \\
\text { bIncludes antidepressants as well as anxiolytics, anticonvulsants/mood stabilizers, antipsychotics, psychostimulants, thyroid hormone (T3), and lithium. } \\
\text { AD = antidepressant; MDD = major depressive disorder; Quan-CCI=Quan-Charlson Comorbidity Index; SD=standard deviation; Std Diff=standardized difference; } \\
\text { TRD = treatment-resistant depression; USD =U.S. dollars. }\end{array}$} \\
\hline
\end{tabular}


FIGURE 1 All-Cause and Physical Condition-Related Health Care Resource Use During Follow-Up Period ( $N=2,317$ per Cohort)

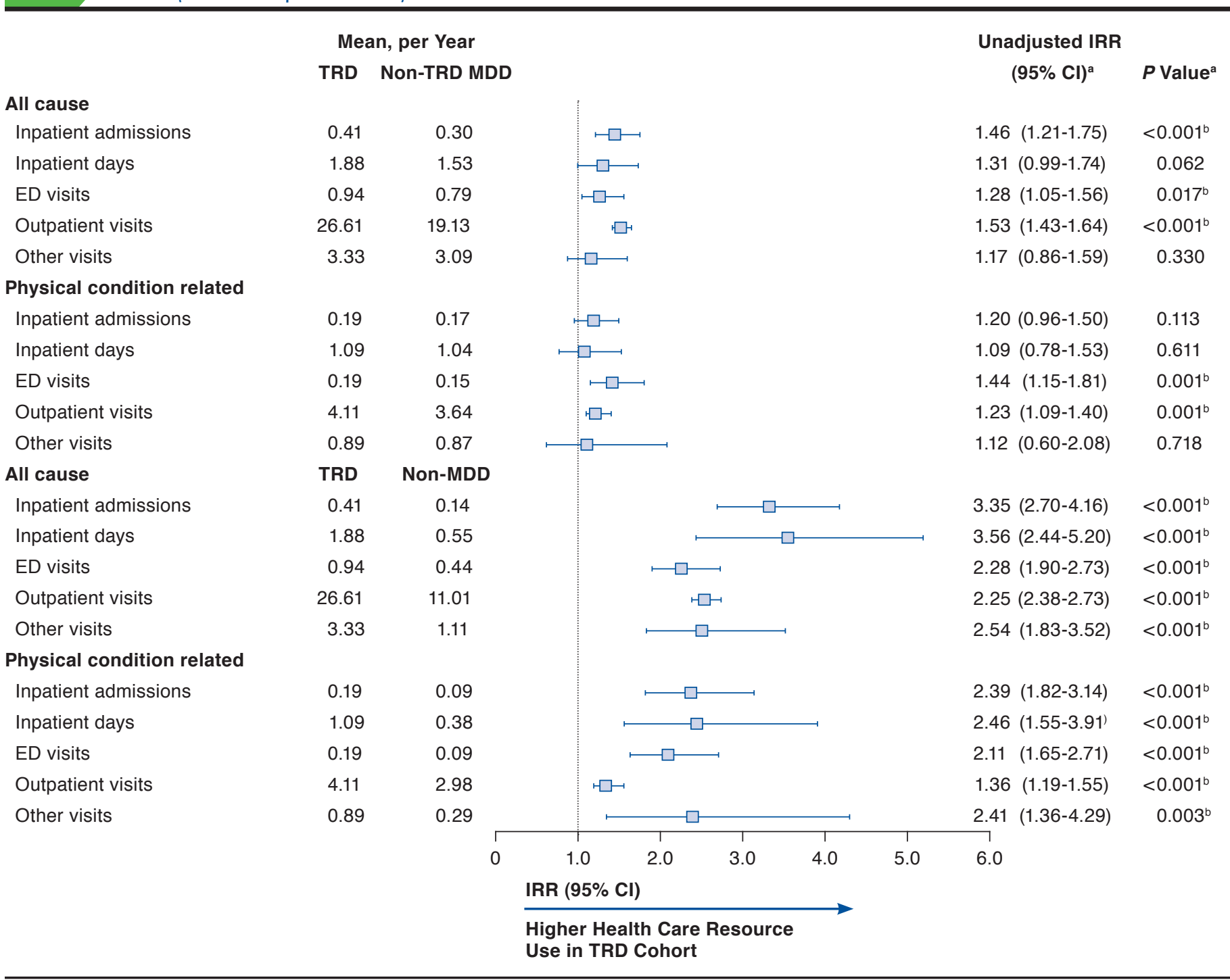

IIRRs, 95\% CIs, and P values were estimated using a generalized linear model with a negative binomial distribution to account for overdispersion.

bignificant at the $5 \%$ level.

$C I=$ confidence interval; $E D=$ emergency department; $I R R=$ incidence rate ratio; $M D D=$ major depressive disorder; $T R D=$ treatment-resistant depression

SUD, 5.4\%) and non-MDD cohorts (anxiety, 1.9\%; SUD, 1.6\%). Among patients with TRD, 69.0\% used a selective serotonin reuptake inhibitor, $31.5 \%$ a norepinephrine-dopamine reuptake inhibitor, $30.6 \%$ a serotonin-norepinephrine reuptake inhibitor, $19.0 \%$ a serotonin modulator, $9.5 \%$ tricyclics or tetracyclics, and $8.1 \%$ norepinephrine-serotonin modulators during the baseline period. A total of 278 (12.0\%) patients in each cohort had work loss data available and were included in the analysis of work loss outcomes. The mean duration of follow-up was 19.7 months in patients with TRD, 20.5 months in patients with non-TRD MDD, and 19.8 months in patients without MDD; the median was 24 months in all cohorts.

\section{Health Care Resource Utilization}

Relative to patients with non-TRD MDD, those with TRD had higher all-cause HRU. Specifically, patients with TRD annually had 46\% more inpatient admissions, 28\% more ED visits, and 53\% more outpatient visits than those with non-TRD MDD (all $P<0.05$; Figure 1). With respect to physical condition-related HRU, patients with TRD annually had 44\% more ED visits and 
FIGURE 2 Mean Annual All-Cause Health Care Costs (2017 USD) During Follow-Up Perioda,b

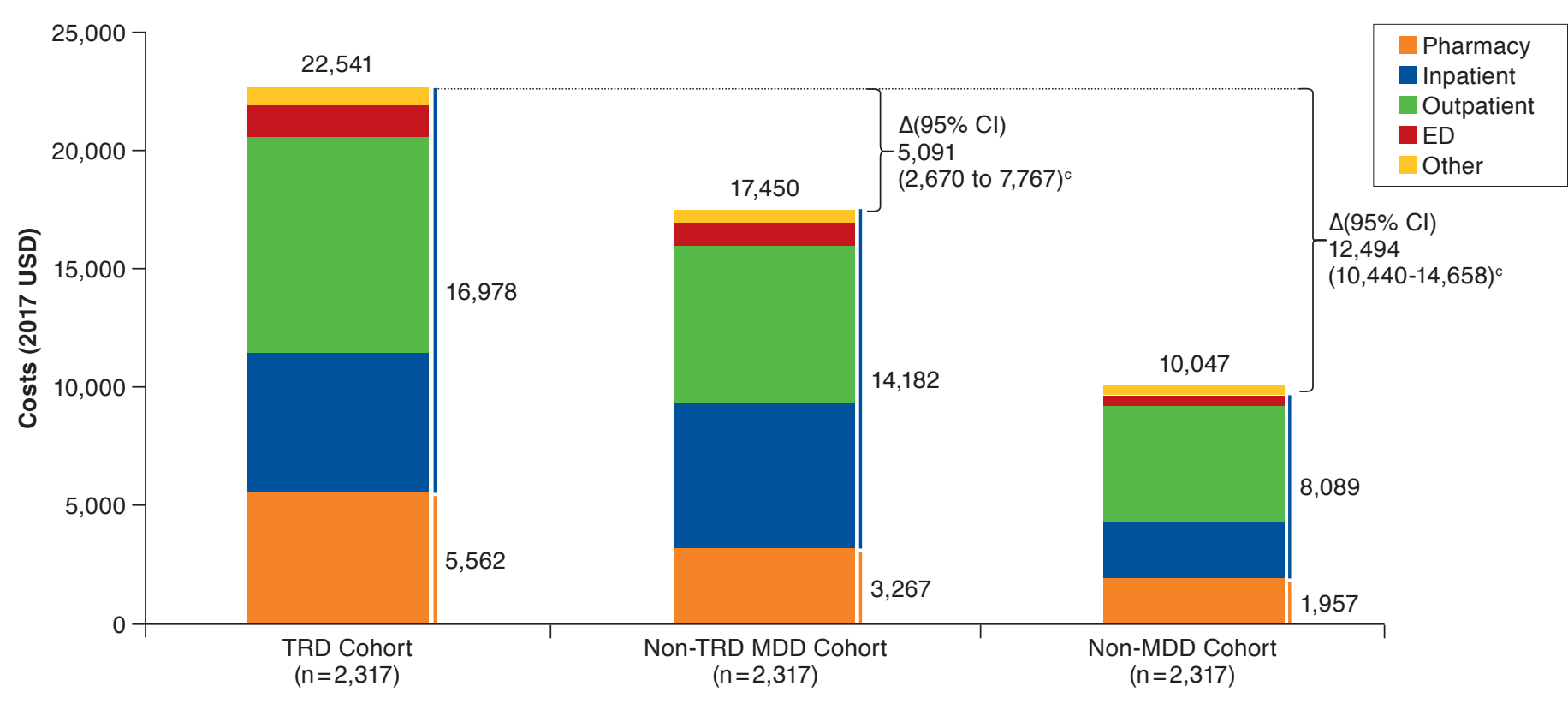

a Health care costs measured from the index date up to 2 years post-index date.

${ }^{b}$ Mean cost differences were estimated using an unadjusted ordinary least squares regression model, and 95\% CIs and P values were estimated using a nonparametric bootstrap procedure $(r=500)$.

cSignificant at the $5 \%$ level.

$C I=$ confidence interval; $E D=$ emergency department $\mathrm{MDD}=$ major depressive disorder; $T R D=$ treatment-resistant depression; USD =U.S. dollars.

23\% more outpatient visits than patients with non-TRD MDD (all $P<0.01$ ).

When using the non-MDD cohort as comparator, the TRD cohort annually had 3.4 times more all-cause inpatient admissions, 3.6 times more all-cause inpatient days, 2.3 times more all-cause ED visits, 2.6 times more all-cause outpatient visits, and 2.5 times more all-cause other visits (all $P<0.01$ ). The TRD cohort also had higher physical condition-related HRU in all categories, including 2.4 times more inpatient admissions, 2.5 times more inpatient days, 2.1 times more ED visits, 1.4 times more outpatient visits, and 2.4 times more other visits (all $P<0.01$ ).

\section{Health Care Costs}

Relative to patients with non-TRD MDD, patients with TRD incurred \$5,091 higher all-cause health care costs annually $(P<0.01)$. This difference was predominantly driven by $\$ 2,423$ higher outpatient and $\$ 2,295$ higher pharmacy costs (all $P<0.01$; Figure 2). Physical condition-related medical costs were similar between the TRD and non-TRD MDD cohorts (Figure 3). About 55\% of the difference in all-cause health care costs between these 2 cohorts was due to higher behavioral health-related costs in the TRD cohort (mean: TRD, \$4,270; non-TRD MDD, \$1,458; $P<0.01$ ).
Relative to patients in the non-MDD cohort, those in the TRD cohort had $\$ 12,494$ higher all-cause health care costs annually $(P<0.001)$, a difference mainly driven by $\$ 4,280$ higher outpatient costs, $\$ 3,605$ higher pharmacy costs, and $\$ 3,462$ higher inpatient costs (all $P<0.01$; Figure 2). Physical condition-related medical costs were $\$ 1,121$ higher in the TRD versus non-MDD cohort annually $(P<0.05$; Figure 3$)$. About $34 \%$ of the difference in all-cause health care costs between the 2 cohorts was due to higher behavioral health-related costs in the TRD cohort (mean: TRD, \$4,270; non-MDD, \$69; $P<0.01$ ), and about $9 \%$ due to higher physical condition-related medical costs.

\section{Work Loss Days}

Patients with TRD had twice as much all-cause work loss annually $(P<0.01)$, including $31 \%$ more medical-related absenteeism and 2.5 times more disability time than those with non-TRD MDD (all $P<0.01$ ); physical condition-related work loss was similar between both cohorts (Figure 4).

Compared with patients without MDD, those with TRD incurred 2.9 times more all-cause work loss annually $(P<0.01)$, including 2.2 times more medical-related absenteeism and 3.3 times more disability time (all $P<0.01$ ); physical conditionrelated work loss was similar between cohorts. 


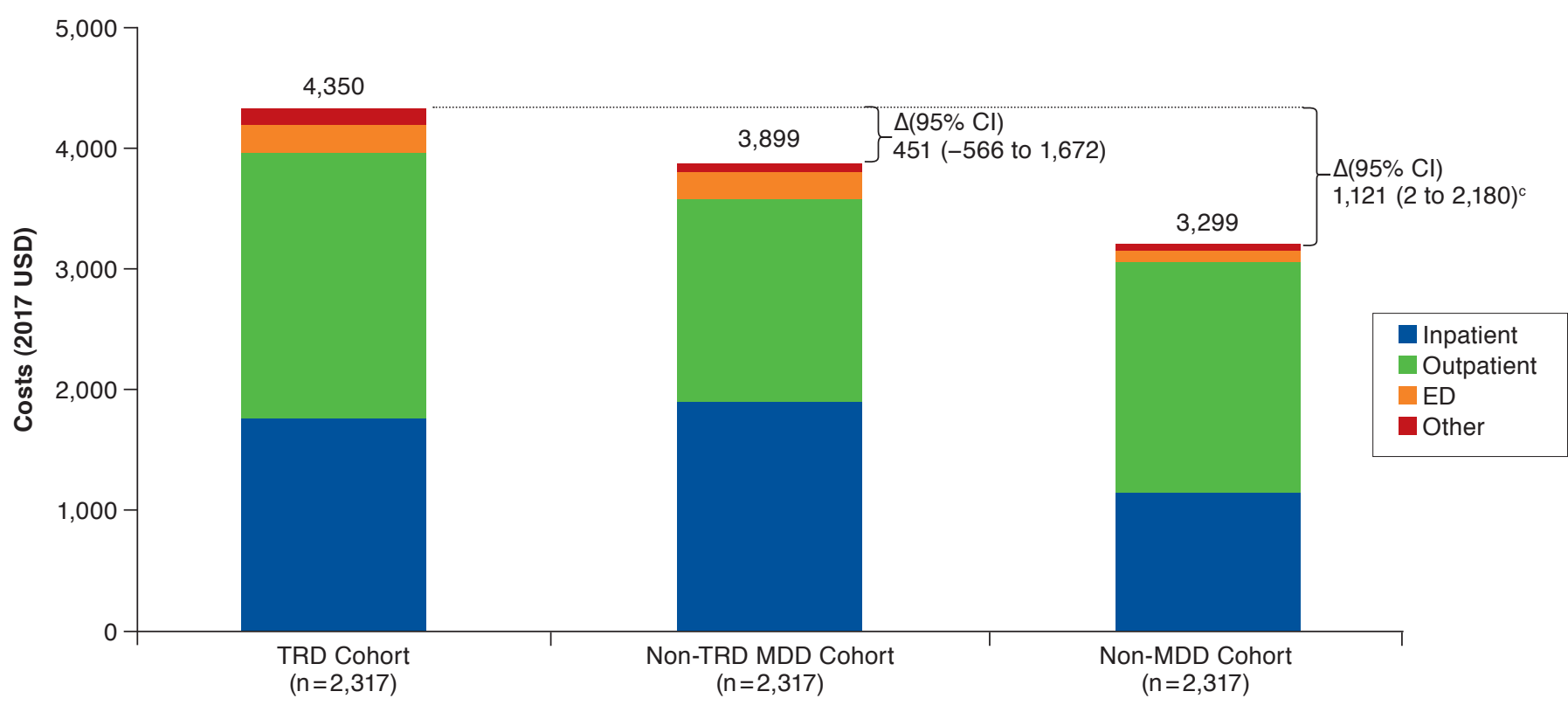

${ }^{a}$ Health care costs measured from the index date up to 2 years post-index date.

${ }^{b}$ Mean cost differences were estimated using an unadjusted ordinary least squares regression model, and $95 \%$ CIs and P values were estimated using a nonparametric bootstrap procedure $(r=500)$.

cSignificant at the $5 \%$ level.

$C I=$ confidence interval; $E D=$ emergency department $\mathrm{MDD}=$ major depressive disorder; $T R D=$ treatment-resistant depression; USD =U.S. dollars.

\section{Costs Related to Work Loss}

Patients with TRD had $\$ 8,676$ higher all-cause work loss costs annually, relative to patients with non-TRD MDD, a difference driven by $\$ 7,487$ higher disability costs (all $P<0.01$; Appendix A, available in online article); medical-related absenteeism costs were not significantly different between cohorts. About $47 \%$ of all-cause work loss cost difference was due to higher behavioral health-related costs in the TRD cohort (mean: TRD, \$5,502; non-TRD MDD, \$1,392; $P<0.01$ ). Physical condition-related work loss costs were similar between cohorts (Appendix B, available in online article).

Relative to patients without MDD, those with TRD had $\$ 10,323$ higher all-cause work loss costs annually $(P<0.01$; Appendix A), including $\$ 7,955$ higher disability costs and $\$ 2,367$ higher medical-related absenteeism costs $(P<0.01)$. About $48 \%$ of all-cause work loss cost difference was due to higher behavioral health-related costs in the TRD cohort (means: TRD, \$5,502; non-TRD MDD, \$521; $P<0.01$ ). Physical condition-related work loss costs were similar between these cohorts (Appendix B).

\section{Discussion}

In this real-world observational study, the economic burden of TRD was assessed in a population of patients with the following physical conditions: cardiovascular, metabolic, respiratory diseases and cancer.

In this population, all-cause health care costs of patients with TRD were 30\% higher than those of patients with non-TRD MDD and more than double those of patients without MDD. Physical condition-related health care costs were similar between patients with TRD and non-TRD MDD. Higher physical condition-related health care costs in the TRD cohort nonetheless explained 9\% of the difference in all-cause health care costs between the TRD and non-MDD cohort. Fifty-five percent of the difference in all-cause health care costs between the TRD and non-TRD MDD cohorts was due to higher behavioral health-related costs in the TRD cohort; this proportion was 34\% for TRD versus non-MDD comparison.

Mean all-cause health care costs observed in the present study among patients with TRD were high. Specifically, these were about 30\% higher than costs reported for the same cohorts in a previous study in commercially insured patients selected regardless of the presence of physical conditions. ${ }^{5}$ Higher mean costs in the current study may reflect the additional costs 
FIGURE 4 All-Cause and Physical Condition-Related Work Loss (Days) During Follow-Up Period $\left(\mathrm{N}=278\right.$ per Cohort) ${ }^{\mathrm{a}}$

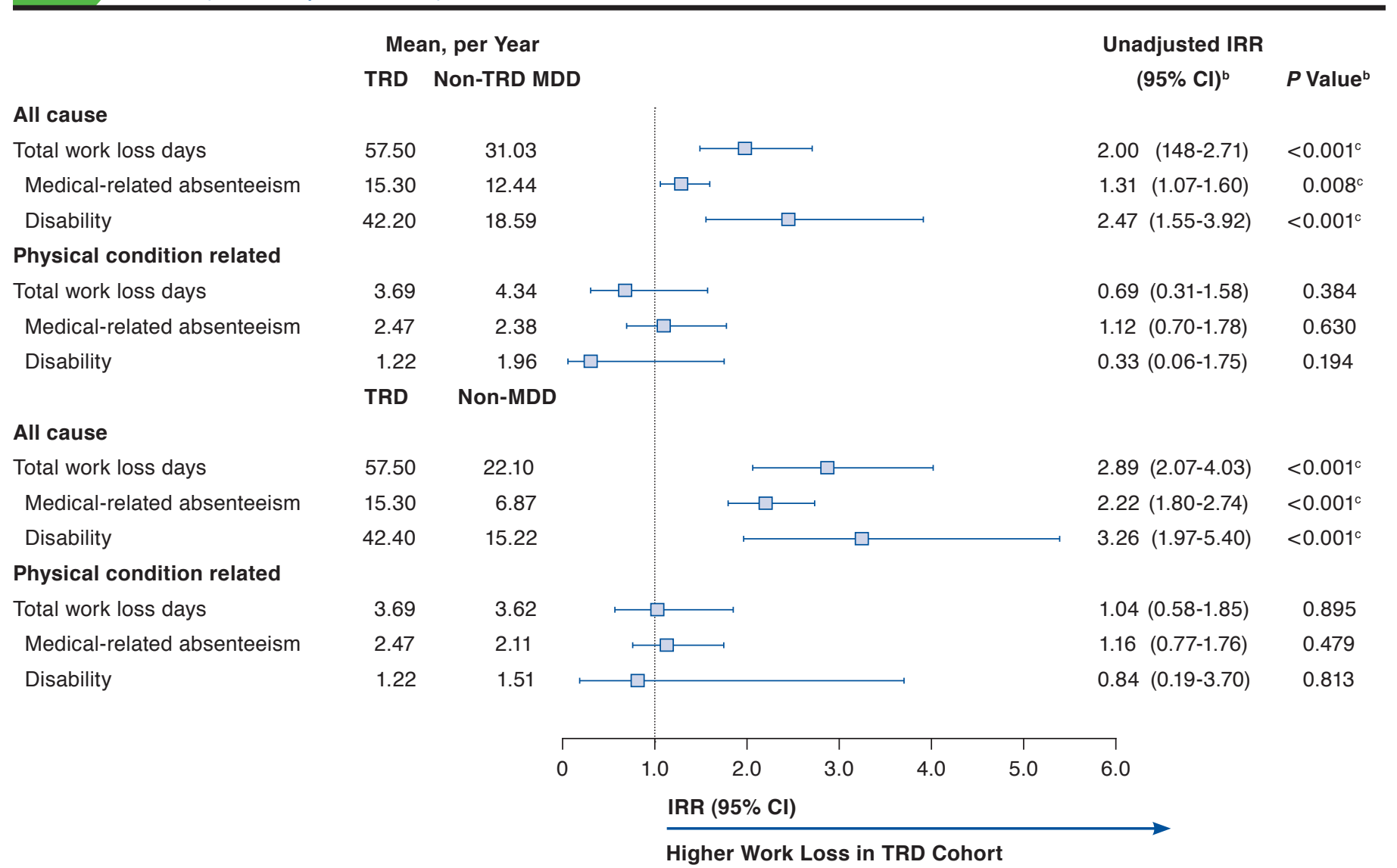

aWork loss measured from the index date up to 2 years post-index date among employees with available work loss data $(n=278)$.

IIRRs, 95\% CIs, and P values were estimated using a generalized linear model with a negative binomial distribution to account for overdispersion.

cSignificant at the 5\% level.

$C I=$ confidence interval; $I R R=$ incidence rate ratio; $M D D=$ major depressive disorder; $T R D=$ treatment-resistant depression.

of physical conditions, although differences in study design (particularly, the index date definition) may also contribute to them. Indeed, the index date of the TRD cohort corresponded to the initiation of a third antidepressant in the current study, whereas Amos et al. (2018) defined it as the initiation of the first antidepressant. ${ }^{5}$ The cost differences between the TRD and non-TRD MDD cohorts (present study, \$5,091; Amos et al., $\$ 7,471$ ), as well as between the TRD versus non-MDD cohorts (present study, \$12,494; Amos et al., \$12,479) were comparable in both studies. ${ }^{5}$ This suggests that the interaction of TRD and physical conditions might not substantially change the overall burden of TRD.

The interplay between co-occurring physical conditions and MDD is complex and is not fully understood. Some indirect evidence from the present study suggest that TRD or MDD might negatively affect the course or management of preexisting physical conditions. Specifically, HRU and total health care costs related to physical conditions were higher among patients with TRD relative to patients without MDD. Although no statistically significant difference in health care costs related to physical conditions was found between the TRD and non-TRD MDD cohorts, patients with TRD had more ED visits due to physical conditions relative to patients with non-TRD MDD. MDD might complicate management of physical conditions or have a negative impact on their course through various channels. For example, patients with mental illness have been reported to have inadequate access to health care. ${ }^{20}$ Moreover, the use of certain antidepressants or adjunctive treatments may play a role as well. Specifically, potential drug-drug interactions may hinder the optimal treatment of certain physical conditions. For safety reasons the use of some antidepressants requires additional monitoring in patients 
with MDD presenting with specific comorbidities, ${ }^{19}$ thereby also increasing HRU and costs. Further research is required to explore the relationship between TRD and the severity of physical conditions.

Previous studies have shown that co-occurring physical conditions are associated with lower rates of response to antidepressants. ${ }^{10,11}$ Although this body of evidence suggests that physical conditions may in turn contribute to TRD, data from the current study are insufficient to further substantiate this hypothesis.

With respect to the indirect economic burden among patients with physical conditions, patients with TRD in this study had all-cause work loss-related costs more than double those of patients with non-TRD MDD and triple those of patients without MDD. Work loss costs related to physical conditions were similar between the cohorts, and higher behavioral healthrelated costs in the TRD cohort explained just less than $50 \%$ of the difference in all-cause work loss-related costs between the cohorts. Interestingly, the incremental work loss-related costs of TRD were not dramatically different when using patients with non-TRD MDD or without MDD as a comparison basis. This seems to suggest that the majority of indirect cost burden related to work loss among medically treated patients with MDD is driven by TRD rather than by non-TRD MDD.

Overall, the results from the current study provide evidence that TRD is associated with substantial economic burden in patients with physical conditions.

\section{Limitations}

The present study is subject to several limitations. First, the algorithm used to identify patients with TRD relied solely on pharmacy claims, and clinical considerations to assess absence of response could not be incorporated. Although the most common definition of TRD was used (i.e., no response to $\geq 2$ antidepressant treatment courses), ${ }^{4}$ there is no consensus definition endorsed by clinical guidelines.

Second, patients included in the non-TRD MDD cohort may have been in remission at the time of their randomly imputed index date, which may have impacted the magnitude of differences in HRU, costs, and work loss-related outcomes. Third, although exact and propensity score-based matching techniques were used to minimize the potential confounding, comparisons may be subject to residual biases due to unmeasured patient characteristics.

Fourth, for patients with less than 1 year of follow-up, annual costs were extrapolated to 1 year based on available data. Fifth, available work loss-related data did not capture presenteeism. Sixth, results of this study might not be generalizable to patients covered by other types of insurance plans (e.g., Medicare, Medicaid) or the uninsured; results in patients with work loss information available might not be generalizable to all patients with commercial insurance plans.
Seventh, the requirement of at least 1 claim with a diagnosis for physical condition might have resulted in the inclusion of some patients with a rule-out diagnosis on a claim. In this case, the levels of reported HRU and costs in all cohorts might be underestimated. Lastly, as with all claims-based studies, analyses are subject to the possibility of inherent limitations related to inaccuracies due to coding errors and missing data.

\section{Conclusions}

In patients with physical conditions, those who additionally had TRD incurred higher HRU and health care costs relative to patients with non-TRD MDD or without MDD. Moreover, patients with TRD incurred higher medical costs related to physical conditions compared with patients without MDD, which suggests that presence of TRD might complicate clinical management of physical conditions or have a negative impact on the course of the disease. TRD was also associated with substantially higher work loss and related costs. Notably, in the TRD cohort, work loss-related costs were more than 2-fold higher versus non-TRD MDD cohort and 3-fold higher versus non-MDD cohort.

\section{Authors}

MARYIA ZHDANAVA, MA; DOMINIC PILON, MA; CARMINE ROSSI, PhD; LAURA MORRISON, MSc; and PATRICK LEFEBVRE, MA, Analysis Group, Montréal, Canada. HARSH KUVADIA, MA, Integrated Resources, Edison, New Jersey. KRUTI JOSHI, MPH, and ELLA DALY, MD, Janssen Scientific Affairs, Titusville, New Jersey. CRAIG NELSON, MD, University of California, San Francisco.

AUTHOR CORRESPONDENCE: Maryia Zhdanava, MA, Manager, Analysis Group, 1190 avenue des Canadiens-de-Montréal, Deloitte Tower, Ste. 1500, Montreal, 2C, H3B 0G7. Tel.: 514.394.4469; Email: Masha.Zhdanava@analysisgroup.com.

\section{DISCLOSURES}

This study was sponsored by Janssen Scientific Affairs (JSA), which was involved in all aspects of the research, including the design of the study; the collection, analysis, and interpretation of data; writing of the report; and the decision to submit the report for publication. Joshi and Daly are employed by JSA. Zhdanava, Pilon, Rossi, Morrison, and Lefebvre are employees of Analysis Group, which received funding from JSA for conducting this study and has received consulting fees from Novartis Pharmaceuticals and GSK unrelated to this study. Kuvadia is employed by Integrated Resources, which has provided research services to JSA unrelated to this study; Joshi reports past employment by and stock ownership in Johnson \& Johnson; Nelson reports advisory board, data and safety monitoring board, and consulting fees from Assurex, Eisai, FSV-7, JSA, Lundbeck, Otsuka, and Sunovion and royalties from UpToDate, unrelated to this study.

This work was presented at AMCP Nexus 2019, held in National Harbor, MD, from October 29 to November 1, 2019 


\section{ACKNOWLEDGMENTS}

Writing assistance was provided by Samuel Rochette, an employee of Analysis Group, which provided paid consulting services to JSA for the conduct of this study.

\section{REFERENCES}

1. Substance Abuse and Mental Health Services Administration. Key substance use and mental health indicators in the United States: results from the 2018 National Survey on Drug Use and Health. HHS Publication No. PEP19-5068. NSDUH Series H-54. August 2019. Available at: https://www.samhsa.gov/data/sites/default/files/cbhsq-reports/ NSDUHNationalFindingsReport2018/NSDUHNationalFindingsReport2018. pdf. Accessed June 3, 2020.

2. National Institute of Mental Health. Major depression. February 2019. Available at: https://www.nimh.nih.gov/health/statistics/major-defrpression. shtml. Accessed June 3, 2020.

3. Joshi K, Zhdanava M, Pilon D, Lefebvre P, Sheehan J. Health care use and associated cost among patients with treatment-resistant depression across payers: a comprehensive analysis. Poster presented at: 2019 AMCP Annual Meeting; March 25-28, 2019; San Diego, CA. Available at: https://www.jmcp. org/doi/pdf/10.18553/jmcp.2019.25.issue-3-a. Accessed June 10, 2020.

4. Gaynes B, Asher G, Gartlehner G, et al. Definition of treatment-resistant depression in the Medicare population. February 9, 2019. Available at: https://www.ncbi.nlm.nih.gov/books/NBK526366/. Accessed June 3, 2020

5. Amos TB, Tandon N, Lefebvre P, et al. Direct and indirect cost burden and change of employment status in treatment-resistant depression: a matched-cohort study using a U.S. commercial claims database. J Clin Psychiatry. 2018;79(2):17m11725.

6. Pilon D, Joshi K, Sheehan JJ, et al. Burden of treatment-resistant depression in Medicare: a retrospective claims database analysis. PLoS One. 2019;14(10):e0223255

7. Olfson M, Amos TB, Benson C, McRae J, Marcus SC. Prospective service use and health care costs of Medicaid beneficiaries with treatment-resistant depression. J Manag Care Spec Pharm. 2018;24(3):226-36. Available at: https://www.jmcp.org/doi/10.18553/jmcp.2018.24.3.226.

8. Pilon D, Sheehan JJ, Szukis H, et al. Medicaid spending burden among beneficiaries with treatment-resistant depression. J Comp Eff Res. 2019;8(6):381-92.
9. Ivanova JI, Birnbaum HG, Kidolezi Y, Subramanian G, Khan SA, Stensland MD. Direct and indirect costs of employees with treatment-resistant and non-treatment-resistant major depressive disorder. Curr Med Res Opin. 2010;26(10):2475-84.

10. Iosifescu DV, Nierenberg AA, Alpert JE, et al. The impact of medical comorbidity on acute treatment in major depressive disorder. Am J Psychiatry. 2003;160(12):2122-27.

11. Trivedi MH, Rush AJ, Wisniewski SR, et al. Evaluation of outcomes with citalopram for depression using measurement-based care in STAR*D: implications for clinical practice. Am J Psychiatry. 2006;163(1):28-40.

12. Pilon D, Szukis H, Joshi K, et al. US integrated delivery networks perspective on economic burden of patients with treatment-resistant depression: a retrospective matched-cohort study. Pharmacoecon Open. 2020;4(1):119-31

13. Greenberg P, Corey-Lisle PK, Birnbaum H, Marynchenko M, Claxton A. Economic implications of treatment-resistant depression among employees. Pharmacoeconomics. 2004;22(6):363-73.

14. Kubitz N, Mehra M, Potluri RC, Garg N, Cossrow N. Characterization of treatment resistant depression episodes in a cohort of patients from a U.S. commercial claims database. PLoS One. 2013;8(10):e76882.

15. Rizvi SJ, Grima E, Tan M, et al. Treatment-resistant depression in primary care across Canada. Can J Psychiatry. 2014;59(7):349-57.

16. Parikh RM, Lebowitz BD. Current perspectives in the management of treatment-resistant depression. Dialogues Clin Neurosci. 2004;6(1):53-60.

17. Gaynes BN. Identifying difficult-to-treat depression: differential diagnosis, subtypes, and comorbidities. J Clin Psychiatry. 2009;70(Suppl 6): $10-15$.

18. U.S. Department of Health and Human Services. 45 CFR 46: pre-2018 requirements. February 16, 2016. Available at: https://www.hhs.gov/ohrp/ regulations-and-policy/regulations/45-cfr-46/index.html\#46.10l. Accessed June 3, 2020.

19. Gelenberg AJ, Freeman MP, Markowitz JC; American Psychiatric Association. Practice guidelines for treatment of patients with major depressive disorder. 3rd ed. October 2010. Available at: https:// psychiatryonline.org/pb/assets/raw/sitewide/practice_guidelines/guidelines/ mdd.pdf. Accessed June 3, 2020

20. Firth J, Siddiqi N, Koyanagi A, et al. The Lancet Psychiatry Commission: a blueprint for protecting physical health in people with mental illness. Lancet Psychiatry. 2019;6(8):675-712.

21. Quan H, Sundararajan V, Halfon P, et al. Coding algorithms for defining comorbidities in ICD-9-CM and ICD-10 administrative data. Med Care. 2005;43:1130-39. 


\section{APPENDIX A Mean Annual All-Cause Work Loss-Related Costs (2017 USD) During Follow-Up Perioda,b}

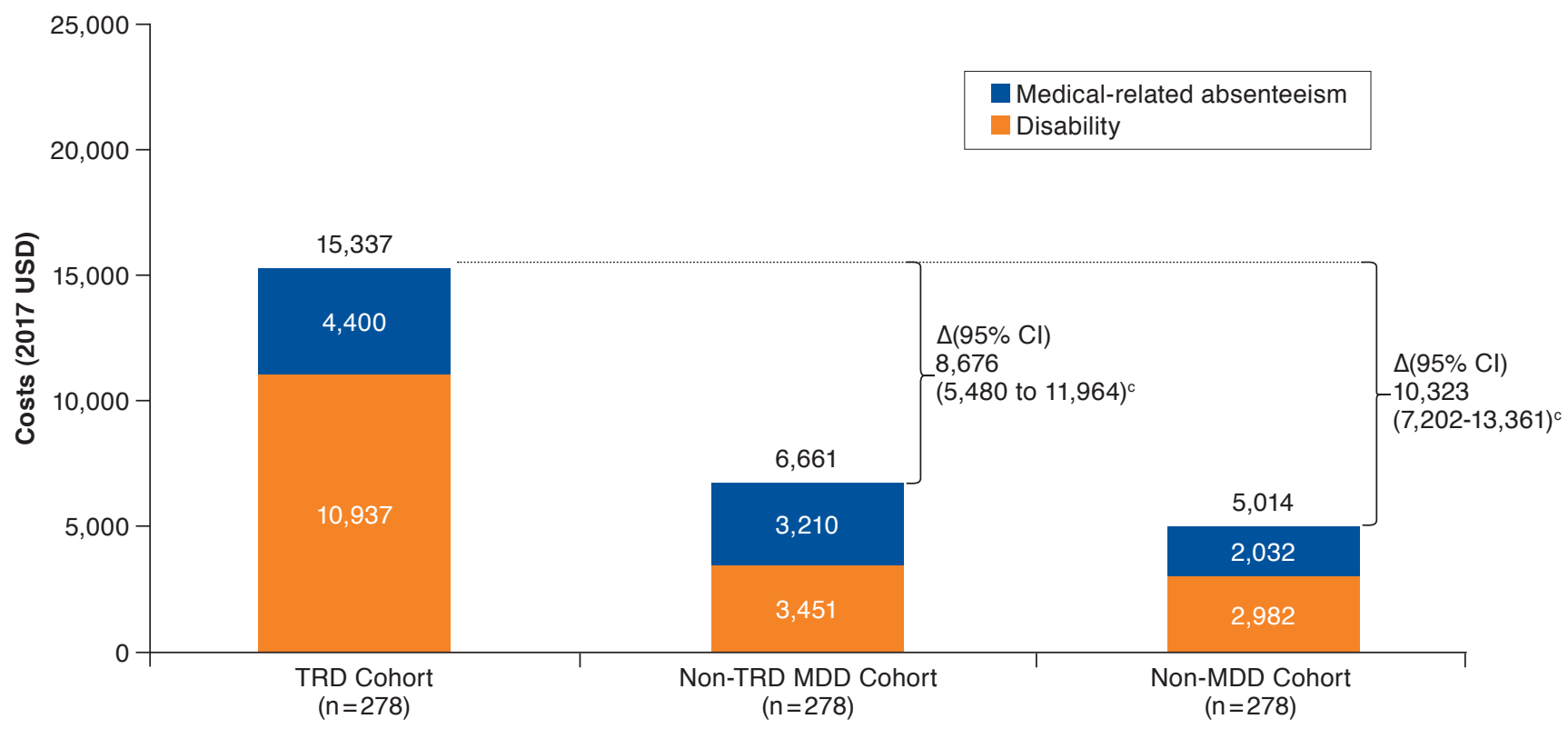

aWork loss-related costs measured from the index date up to 2 years post-index date among employees with available work loss data ( $n=278)$.

${ }^{b}$ Mean cost differences were estimated using an unadjusted ordinary least squares regression model, and 95\% CIs and P values were estimated using a nonparametric bootstrap procedure $(r=500)$.

cSignificant at the 5\% level.

$C I=$ confidence interval; $M D D=$ major depressive disorder $; R D=$ treatment-resistant depression; USD =U.S. dollar. 


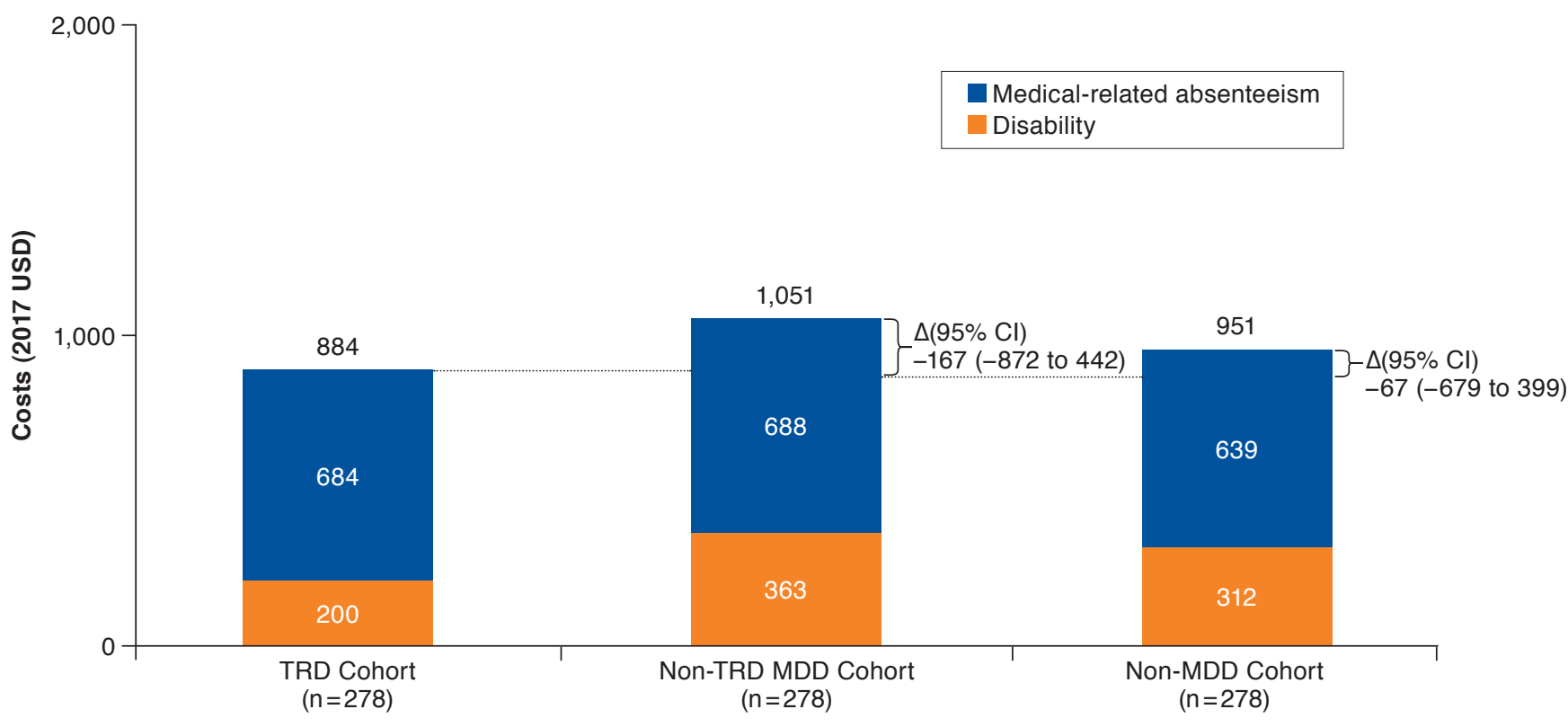

aWork loss-related costs measured from the index date up to 2 years post-index date among employees with available work loss data $(n=278)$.

${ }^{b}$ Mean cost differences were estimated using an unadjusted ordinary least squares regression model and $95 \%$ CIs and P values were estimated using a nonparametric bootstrap procedure $(r=500)$.

$C I=$ confidence interval; $M D D=$ major depressive disorder; $T R D=$ treatment-resistant depression; USD =U.S. dollars. 